\title{
¿Debe la alta función pública parecerse a la sociedad? Algunas enseñanzas de la reforma de la alta función pública en Francia
}

\section{Should the senior civil service resemble society? Some lessons from the reform of the senior civil service in France}

\author{
Jorge Crespo González \\ Universidad Complutense de Madrid (España) \\ ORCID: https://orcid.org/0000-0002-5451-6489 \\ jorge.crespo@cps.ucm.es
}

\section{NOTA BIOGRÁFICA}

Profesor de Ciencia Política y de la Administración en la Universidad Complutense de Madrid (UCM). En la actualidad además es coordinador del área de Función Pública Estratégica del Instituto Complutense de Ciencia de la Administración. Sus intereses científicos pivotan sobre la gobernanza y la gestión multinivel, función pública estratégica, función directiva y gestión de personas en las Administraciones públicas.

\section{RESUMEN}

El presente articulo versa sobre la alta función pública a la luz de la reforma de la misma que en la actualidad se desarrolla en Francia, extrayendo las enseñanzas pertinentes para el caso español. El análisis se centra en responder a las siguientes preguntas de investigación: ¿debe la alta función pública parecerse a la sociedad a la que sirve? y ¿en qué medida la reforma francesa desprende buenas prácticas importables a nuestra alta función pública? Y todo ello enmarcado en la hipótesis de que una sesgada selección de altos funcionarios representa no solo un problema técnico, sino de democracia. Uno de los principales hallazgos del trabajo sugiere la importancia de la alta función pública no solo por lo que hace, sino como instrumento para que los ciudadanos, con independencia de sus recursos, puedan tener un acceso equitativo a las funciones políticas, dada la interpenetración de la alta burocracia con la política y los políticos.

\section{PALABRAS CLAVE}

Alta función pública; política; representatividad; inclusión; España; Francia; reforma.

\begin{abstract}
This article aims to analyse the reform of the French hight public officials (haute fonction publique) that it is becaming since President Macron arrive to power. extracting the pertinent lessons for the Spanish case. The analysis focuses on answering the following research questions: first, should hight public officials resemble the society that they serves?; and, second, the aforementioned French reform provides some good practices for Spanish high public officials (alta función pública)? Finally, it will traited the following hypothese: the selection of senior officials represents not only a technical problem, but also a democracy one. One of the main findings of the work suggests the importance of the high public function, not only for what it does, but also as an instrument that citizens, regardless of their resources, can use to have an equitable access to political functions, given the interpenetration of the high bureaucracy with politics and politicians.
\end{abstract}

\section{KEYWORDS}

Public Officials; Senior Civil Service; politics; representativeness; inclusion; Spain; France; reform. 


\begin{abstract}
SUMARIO
INTRODUCCIÓN. 1. QUÉ ES LA ALTA FUNCIÓN PÚBLICA Y QUÉ FUNCIONES TIENE. 2. PODER DE LA ALTA FUNCIÓN PÚBLICA Y PERSONAL POLÍTICO. 3. LA ALTA FUNCIÓN PÚBLICA COMO PROBLEMA INSTITUCIONAL/DEMOCRÁTICO. 4. LOS SESGOS EXISTENTES EN LA COMPOSICIÓN DE LA ALTA FUNCIÓN PÚBLICA ESPAÑOLA. 5. LA EXPERIENCIA FRANCESA: LA REFORMA DE LA LEY DE MAYO DE 2019 O LA SUPRESIÓN DE LA ENA. a) CONTEXTO Y DIAGNÓSTICO. b) LA SITUACIÓN DE LA ENA. c) LAS PROPUESTAS. 1. Diversificar el reclutamiento y la selección. 2. La descompartimentación de la alta función pública. 3. Dinamizar las carreras de los altos funcionarios. 6. ENSEÑANZAS PARA EL CASO ESPAÑOL. 7. CONCLUSIONES. REFERENCIAS BIBLIOGRÁFICAS.
\end{abstract}

\title{
INTRODUCCIÓN
}

En los últimos años se está produciendo un debate sobre las relaciones entre sociedad y Administración Pública, que se pretenden inclusivas y armónicas. Un instrumento para verificar el grado de apertura de la Administración es el análisis de la composición de su función pública, y más en concreto del segmento de empleados públicos que participan decisivamente en la formulación y gestión de las políticas públicas. Un exponente del cambio que se plantea, en lo que concierne el papel de la alta función y su relación con la sociedad de la que es expresión, es el proyectado por el Gobierno francés respecto de la Ecole Nationale d'Administration (ENA). El interés de los cambios que se proponen, junto al carácter ilustrativo que las reformas administrativas francesas acaban teniendo para la Administración española, justifican este artículo.

Hay 2 preguntas de investigación que han alentado este estudio. En primer lugar, ¿debe la alta función pública parecerse a la sociedad a la que sirve? Y, en segundo lugar, ¿qué medidas de las adoptadas en Francia recientemente sobre su alta función pública pueden considerarse buenas prácticas para la nuestra? Con la respuesta a estas preguntas se pretende verificar la hipótesis de que, en determinadas condiciones, una mala o sesgada selección de los altos funcionarios públicos puede devenir en un problema de democracia.

La exposición seguirá el siguiente orden. Primeramente, se planteará una definición de «alta función pública», junto con sus funciones; en segundo lugar, se analizarán las relaciones entre altos funcionarios y políticos, mostrando que se trata de dos colectivos con grandes solapamientos funcionales y subjetivos; posteriormente, se tratará cómo la función pública puede devenir en problema institucional o de democracia; en cuarto lugar, se tratará la reforma en trance de realización sobre la alta función pública francesa, y las enseñanzas para el caso español. Y finalmente se muestran los hallazgos del trabajo en las conclusiones.

\section{QUÉ ES LA ALTA FUNCIÓN PÚBLICA Y QUÉ FUNCIONES TIENE}

En este trabajo, siguiendo el camino marcado por grandes especialistas (por ejemplo, Baena, 1988) se va a identificar la alta función pública con aquellos funcionarios, y cuerpos o colectivos en que se insertan en el ejercicio de su actividad, que ejercitan al máximo nivel, y con altísima especialización, el poder administrativo. Lo que implica, aunque deba introducirse una nota de relativismo ya que las culturas administrativas de los diferentes Estados juegan un papel importante y podrían incluirse aspectos complementarios, que las notas esenciales de los funcionarios que forman parte de la alta función pública radican en su pertenencia a un cuerpo o colectivo, muy especializado, al que se le reservan funciones estratégicas para el Estado, y para cuyo acceso se requiere una titulación de carácter superior. Por lo que puede decirse que profesionalización y especialización están en la base de la alta función pública, pero interpretando esta última no como un único colectivo sino como un elenco o constelación de grupos/cuerpos en constante interacción colaborativa o conflictiva, entre sí y con los políticos.

Sintetizando, la concreción de las notas específicas de la alta función pública en España, y por extensión del resto de países de cultura administrativa napoleónica, incluye (Baena, 1988, pp. 440-445):

a) Organización en Cuerpos o Colectivos.

b) Una altísima competencia y profesionalización.

c) Permanencia protegida en el empleo público (a fin de garantizar su imparcialidad y neutralidad).

d) Capacidad de frenar decisiones políticas o veto decisorio.

e) Capacidad de obstaculizar la aplicación de las políticas (veto paralizante). 
f) Disponer como instrumentos de poder, además de la estabilidad en el empleo y su posición jerárquica, su influencia en la legislación, el control de los recursos y la cercanía con el poder decisorio público y privado.

g) Tendencia con carácter general a no presentarse como «mero» funcionario, identificándose más con las competencias (generales o especiales) de su cuerpo.

h) Participación en órganos de cooperación/colaboración/coordinación intergubernamentales o multinivel (Crespo, 2017).

Esa noción tiene la virtud de ser coherente con la que mantiene la OCDE (2009, p. 118; 2018, pp. 4-51): «un porcentaje muy pequeño de todos los funcionarios del gobierno central, la mayoría no nombrados políticamente [...] que constituyen el escalón entre los políticos y la Administración pública ....son responsables de la implementación de los instrumentos legales y de las estrategias políticas, y de la coherencia y eficiencia de las actividades del gobierno», y por todo ello un instrumento clave de la gobernanza pública.

Además, permite excluir de su perímetro a colectivos funcionariales que no ejercen al máximo nivel el poder administrativo, al personal político o de designación política (aunque pueda estar integrado con frecuencia por altos funcionarios) e, incluso, a la función pública directiva, aunque ésta se solape en muchos casos orgánica y funcionalmente con los colectivos de la alta función pública.

Con carácter general, tienen carácter de altos funcionarios: la alta jerarquía militar, los jueces, el cuerpo diplomático, los letrados del Estado y del resto de instituciones, el cuerpo generalista especializado en Administración y gestión pública, entre otros (Baena, 1988, pp. 438 y ss.).

\section{PODER DE LA ALTA FUNCIÓN PÚBLICA Y PERSONAL POLÍTICO}

Los teóricos de la Administración Pública se han esforzado en conocer la raíz del poder que los altos funcionarios desprenden tanto de cara a la sociedad como en relación con los políticos electos. Uno de los que más ha avanzado en la determinación de las fuentes del poder burocrático, o de la alta función pública, ha sido Panebianco.

Panebianco (1995) establece que el colectivo de los políticos dispone de una fuente de poder insoslayable: el respaldo de haber sido elegidos en procesos electorales y, por ello, al menos en escenarios democráticos, asumir las funciones de gobierno. Sin embargo, a pesar de esta fortaleza y la legitimidad que otorgan las urnas, y dando por descontada su capacidad para generar un relato de lo que necesita la sociedad y su proyección en el tiempo, respecto de los altos funcionarios suelen carecer de ciertos recursos (cognitivos, organizativos, normativos, técnicos, etc.) que suavizan su capacidad de mando sobre ellos, y que invitan a apoyarse especialmente en la expertise de los altos funcionarios. Los altos funcionarios, por su parte, no cuentan con ese respaldo electoral de base, pero disponen de unas fuentes de poder evidentes: grandes conocimientos técnicos, estabilidad en el empleo público, uso (a veces opaco) de los instrumentos y procedimientos administrativos, capacidad para movilizar a sus clientelas administrativas, generación si fuera necesario de discursos o argumentaciones de combate rebozados de un barniz de interés público si vieran amenazada su posición. Estas fuentes de poder permiten a la alta función pública asumir un rol cercano al político pero sin responder públicamente de sus decisiones y acciones.

Es cierto que ese poder de la alta función pública no es monolítico. Ya se refirió anteriormente que la cultura administrativa nacional y el contexto político y social de cada Estado tiene una gran relevancia, por lo que hay aspectos que permiten incrementar el grado de penetración del poder burocrático en la política y, viceversa, otros que permiten un mejor dominio de la alta función pública por los políticos. Entre los aspectos contingentes que pueden actuar como intensificadores del poder de la alta función pública, se encuentra el grado de politización del asunto a tratar (a mayor politización, menor poder de la alta función pública y mayor de otros actores), el grado de complejidad técnica o normativa (a mayor complejidad, mayor poder de la alta función pública), el grado de integración y cohesión de la alta función pública como institución (a mayor integración y cohesión, mayor poder de la alta función pública) y, como contrapartida, el grado de integración y cohesión del gobierno y del entramado político-institucional (a mayor integración y cohesión, menor poder de la alta función pública) (Panebianco, 1995, p. 396).

1 Puede consultarse en: "Recomendación del Consejo sobre capacidad y liderazgo de la función pública". https://www.oecd.org/ gov/pem/recomendacion-del-consejo-sobre-liderazgo-y-capacidad-en-la-funcon-publica.pdf (fecha de acceso: 30 de junio de 2021). 


\section{LA ALTA FUNCIÓN PÚBLICA COMO PROBLEMA INSTITUCIONAL/DEMOCRÁTICO}

Teniendo en cuenta la consideración de élite que generalmente se otorga a la alta función pública, por lo que hace, por las relaciones que establece, su origen social, su proximidad al ámbito de decisión político y por el solapamiento entre los dos colectivos, la cuestión de quiénes son los altos funcionarios del Estado, cuál es su origen (educativo, social, económico y territorial) y su forma de reclutamiento y selección pasa de constituir un problema meramente técnico a convertirse en un elemento al que prestar atención política y académica, por su relación con la generación de aceptación y confianza en el sistema político, reforzando la legitimidad o lo contrario.

Ya Panebianco (1995, pp. 384 y ss.) nos informaba de que la alta función pública puede tratarse en tanto élite burocrática, exponiendo un elenco de criterios cuyo estudio darían claves para su determinación. Entre ellos, citaba la extracción social, la consideración o prestigio que mantenga en la sociedad, la forma de reclutamiento, socialización y carrera (pudiendo estar ante una élite burocrática cerrada en entrada y salida, élite cerrada en entrada y abierta en salida, y élite abierta en entrada y salida). También señalaba la relación entre generalistas y especialistas dentro de esa misma élite.

En España, Baena et al. (1984, p. 75) defendió que hasta el inicio de la transición, las élites políticas, económicas y administrativas españolas tenían un elevado nivel de integración, respecto de la cual la burocracia (entendida como cuerpos de funcionarios superiores que ocupaban puestos en las Cortes, Consejos de Administración de grandes empresas, el Gobierno y la Administración) asumía una gran responsabilidad. Asimismo, reconoció que la presencia de burócratas en esa élite era de gran entidad. Por otra parte, este autor destacó la importancia de la élite administrativa en la confección de la cultura política democrática y la conformación del liderazgo y entramado de cargos políticos, así como las ramificaciones exteriores al sector público concluyendo que de ella se nutren las élites, en general, del país. Lo que le llevó a desarrollar una larga investigación, de carácter empírico, sobre las élites y los grupos de poder en España (Baena, 1999). Finalmente, este autor expresa la importancia de analizar, en el estudio de la alta función pública, la tradición familiar, la endogamia y la procedencia social, junto a su relación con los grupos de poder (Baena, 1988, pp. 446 y ss.).

En definitiva, el análisis de las élites administrativas constituye un instrumento esencial para estudiar cómo el poder público ejerce la dominación en la sociedad a través de la Administración y políticos y empleados públicos, recalcando el carácter imprescindible del análisis sobre el complejo de relaciones en el que intervienen y en el que se integran, así como de la gestión de los medios para conseguir los fines públicos.

Por todo ello, cabe concluir que por sus funciones, cercanía del poder político del que con frecuencia forman parte, como por su poder ad extra para integrar las élites del Estado, así como por participar decisivamente en la conformación de la cultura y legitimidad políticas, se trata de un objeto de estudio de alto interés. Se debe insistir en sus funciones, en las que destaca su participación en las políticas públicas (formulación, implantación y evaluación) pudiendo generar posibles vetos, tanto decisorios como ejecutorios. También deben recordarse las labores de auxilio y asesoramiento a los políticos y las funciones intergubernamentales que realizan. Pero además de ello, y por si no fuera suficiente, hay un aspecto adicional que ubica la reflexión un escalón por encima de lo que hasta ahora hemos referido. Se trata del solapamiento subjetivo entre los integrantes de un colectivo y otro, es decir, entre los altos funcionarios respecto de los políticos y viceversa. En otras palabras, los datos de diferentes investigaciones (por ejemplo, Rodriguez Teruel, 2011 y 2019), trasladan una alta correlación entre la pertenencia a un colectivo de la alta función pública y la asunción de responsabilidades políticas en las diferentes instancias del poder ejecutivo, legislativo y, cómo no, judicial ${ }^{2}$. Lo que ocurriría, entre otras causas, porque en el desempeño profesional adquieren habilidades necesarias para la política, ofrecen flexibilidad en el desarrollo de la carrera y, además, la obtención con frecuencia de un contacto previo con las élites sociales y redes de conocimiento que facilitan el acceso al ámbito político (Rodríguez Teruel et al. 2019, pp. 592-593). Siendo esto así, ser alto funcionario otorgaría un acceso privilegiado a la función política, lo que haría que el problema de selección de las élites administrativas no constituyera solo una cuestión técnica u organizativa con ribetes institucionales, sino que se adentraría en un territorio más complejo, puesto que se trataría de un problema de democracia, al proponerse como un escalón que funciona como una suerte de acelerador o ascensor hacia el mundo de la política, que estaría reservado a unos pocos que, de acuerdo con los estudios sociológicos, proceden de ambientes acomoda-

2 Por ejemplo, Rodríguez Teruel et al. (2019, p. 595) indican que desde 1982 hasta la 2019 en torno al $65 \%$ de los miembros del gobierno nacional tenían la condición de funcionarios. 
dos, cultivados y con diferentes sesgos socioeconómicos y territoriales. Esta aseveración, especialmente sensible para la ciudadanía en momentos de crisis, plantea que si bien en algún momento del pasado los sesgos eran explicables, en nuestros días se ha convertido en el talón de Aquiles de las funciones públicas de diversos países, especialmente aquellos que disponen de funciones públicas cerradas próximas al modelo napoleónico, como es el caso de Francia y España.

$Y$ es en ese marco en que debemos entender, primero, lo importante que es reconocer el problema del estudio de la alta función pública, y en segundo lugar, y habida cuenta su importancia democrática, política e institucional, la necesidad de que se parezca a la sociedad a la que sirve para no trasladar sesgos que hagan que unos ciudadanos tengan un acceso privilegiado a la acción política simplemente por ser altos funcionarios. Pero ¿cuáles son esos sesgos que impiden que la alta función pública se parezca a la sociedad?

\section{LOS SESGOS EXISTENTES EN LA COMPOSICIÓN DE LA ALTA FUNCIÓN PÚBLICA ESPAÑOLA}

Determinados rasgos hacen de los cuerpos que forman parte de la alta función pública una suerte de colectivo aparte que paradójicamente debe operar sobre una realidad social que no siempre conoce bien, puesto que el mismo incorpora un conjunto de sesgos que genera estímulos, percepciones y nociones sobre lo que hay que hacer, o no, que no siempre coincide con las necesidades de sociedad y del sistema político. Sobre esto y otras disfunciones serias de la gestión de personal en el marco de las Administraciones públicas españolas han escrito relevantes autores (veáse por todos Arenilla y Delgado, 2019; o Castillo Blanco, 2020).

Ciertos sesgos o deformaciones de ver la realidad fueron descritos tempranamente por los autores que trataron las disfunciones de la burocracia, y que destacaban aspectos como el desplazamiento de metas (para el funcionario se trata más de crear normas o de seguir el iter normativo y procedimental que de resolver problemas ciudadanos), la incapacidad adiestrada (la formación del funcionario no le fuerza a ver la realidad, por lo que en vez de otorgar capacidad proporciona incapacidad), reforzadas por la psicosis profesional y la despersonalización (Merton, 1964, pp. 275-286).

Algunos de estos sesgos están muy presentes en la reforma propuesta en Francia, pero también cabe recordar otros aspectos de carácter sociológico y que están relacionados con la edad, formación, género, procedencia social y dispersión territorial. Unos y otros van a ponerse en relación, por ahora, con la situación española y especialmente con datos empíricos procedentes del Cuerpo de Administradores Civiles del $E_{\text {Estado }}{ }^{3}$, uno de los cuerpos más importantes de la alta función pública española. De los datos no hay que esperar una validez estadística en términos inferenciales, pero sí un componente indiciario reforzado para anticipar cómo será la situación en otros cuerpos de esa misma alta función pública. Veamos ahora algunos de los sesgos más importantes de la alta función pública española:

a) Una alta función pública muy envejecida. Los datos sobre la función pública de la Administración General del Estado (AGE) en general son elocuentes, puesto que la edad media de los empleados es de 52 años, 10 años superior a la media de la población española. Además de una necesidad de ir anticipando la desvinculación de este personal por jubilación, capitalizando su conocimiento y experiencia, parece lógico pensar que estamos ante generaciones que se han formado, socializado y crecido en un entorno muy distinto al de las nuevas cohortes poblacionales, lo que se muestra en aspectos prácticos tales como la adaptación a las nuevas tecnologías.

b) Una alta función pública no representativa territorialmente. En un estudio (Crespo, 2015) se evidenció cómo la extracción territorial de los integrantes del cuerpo de Administradores Civiles del Estado se nutría especialmente, y con persistencia histórica, de la región madrileña (más que triplicando los miembros que le corresponderían por demografía), y las regiones mesetarias. En los últimos años se había detectado una mejoría de la representación de miembros procedentes de Galicia, Asturias y Aragón; pero sin embargo sorprendía, entre las CC.AA infrarrepresentadas, Cataluña (sobre un tercio de los que le correspondería por peso demográfico), que ya partía de una fuerte infrarrepre-

3 El trabajo empírico incorporó la respuesta a un cuestionario de 102 de sus miembros y la realización de múltiples entrevistas a académicos de la talla de Sabino Cassese, Manuel Arenilla o Mariano Baena del Alcázar. La fase de obtención de datos finalizó a principios de 2017 y debemos agradecer la colaboración de la Asociación Profesional del Cuerpo Superior de Administradores Civiles del Estado y del Instituto Nacional de Administración Pública de España. 
sentación en los años 60 y 70 según los estudios de Álvarez (1980), junto a Extremadura, Navarra y Canarias. Lo relevante de estos datos no es solo mostrar los escasos ecos que ha tenido la descentralización política de los últimos decenios en la procedencia de la alta función pública del Estado, sino indicar que ello puede tener influencia en términos culturales, institucionales y sociales. Una pregunta sería: ¿Por qué los ciudadanos de estas regiones no muestran interés o no aprueban las pruebas de acceso de los altos cuerpos del Estado? Algunas consideraciones sobre el particular pueden consultarse en Crespo (2018).

c) Una alta función pública muy centralizada en Madrid. Se produce una notable centralización de los puestos de trabajo que desarrollan los Altos Cuerpos de la AGE en Madrid. Ello se muestra en que por ejemplo el $84 \%$ de los Administradores Civiles del Estado trabaja en Madrid ${ }^{4}$, o que una altísima proporción de los miembros de la Carrera Diplomática sin destino en el exterior también lo hace.

d) Una alta función pública con altas dosis de reproducción social y que en general procede de las capas acomodadas de la población. De acuerdo con los datos publicados en Ruano et al. (2014), en la alta función pública existe una alta reproducción social, con visos de endogamia cultural, puesto que existe una tendencia reforzada a que los hijos de padres directivos públicos, o privados, o que forman parte de cuadros superiores de la Administración, accedan a la alta función pública. Ello dentro de la tendencia general a que de personas funcionarias salgan hijos que también sean servidores públicos. Obviamente, deben pasar el proceso selectivo correspondiente, pero la orientación, inducción y contactos ofrecidos en casa, junto con el soporte familiar para preparar durante años las pruebas, parecen constituir una palanca eficaz para el éxito en el mismo. En este sentido, una muestra expresiva del carácter clasista de la alta función pública es que, una vez finalizados los estudios superiores, los candidatos destinan de media casi 3 años a la preparación de las pruebas, lo que deben acompañar de aprendizaje o refuerzo de lenguas extranjeras y el pago de academias o preparadores.

e) Una alta función pública con altísima cualificación. En la función pública del Estado es muy frecuente que el grado de formación que aporte el empleado público sea superior al solicitado para el puesto a desempeñar, y en lo referido a los altos funcionarios, que de por sí ya requieren un grado universitario, ocurre que una proporción creciente cuenta con estudios de master universitario y doctorado (Ruano et al., 2014). Esto es coherente con las notas características de la alta función pública, su alta cualificación y profesionalización.

f) Una alta función pública que integra paulatinamente la diversidad de género y de discapacidad. La AGE ha incorporado la diversidad de género de manera más positiva que el conjunto del mercado de trabajo. En efecto, el INE (datos del primer trimestre de $2021^{5}$ ) informa de que la tasa de actividad femenina es para el conjunto de actividades del $52,91 \%$, mientras que la masculina es del $62,74 \%$. En el conjunto de las Administraciones Públicas de España, el boletín estadístico de personal, de carácter trimestral ${ }^{6}$, indica que la tasa de actividad femenina es mayor que la masculina ( $56,7 \%$ vs. $43,3 \%$ ). Para la AGE, dejando fuera las Fuerzas Armadas y los Cuerpos de Seguridad del Estado, se sigue la tendencia general de las Administraciones, siendo la presencia femenina mayor en términos porcentuales $(49,2 \%$ hombres; $50,8 \%$ mujeres). Respecto de la presencia de mujeres en los altos cuerpos, aunque se ha mejorado en los últimos años, le queda recorrido de mejora en los cuerpos especiales mientras que su situación es equitativa en los generales (como por ejemplo, en el de Administradores Civiles del Estado). Y, finalmente, las medidas positivas a favor de facilitar la integración de personas con discapacidad en la Administración Pública (entre otras, la reserva de un porcentaje de plazas para este sector) están dando sus frutos, aproximándose paulatinamente al objetivo del $2 \%$ propuesto para cada Administración, lo que no impide reconocer los pocos estudios existentes sobre el particular y la falta de adaptación de algunos sectores al ingreso de personas con discapacidad.

4 En 2016, por ejemplo, de los 982 funcionarios en servicio activo del Cuerpo Superior de Administradores Civiles del Estado, $824(83,91 \%)$ están destinados en Madrid, 79 en provincias (8,044\%) y $79(8,044 \%)$ en la Administración exterior (embajadas y organismos internacionales). Datos extraídos de http://www.inap.es/que-es-el-cuerpo-superior-de-administradores-civiles-del-estado1 (fecha de acceso: 20 de julio de 2016).

5 https://www.ine.es/jaxiT3/Datos.htm?t=4050\#!tabs-grafico (fecha de acceso: 24 de junio de 2021).

6 http://www.mptfp.es/portal/funcionpublica/funcion-publica/rcp/boletin.html (boletín de julio de 2020. Fecha de acceso: 24 de junio de 2021. 
g) Una Alta función pública cerrada a la realidad sobre el terreno. A pesar de que la edad media de los candidatos aprobados en los altos cuerpos se ha incrementado en los últimos años, y ello les ha permitido en algunos casos disponer de una cierta experiencia laboral previa, aspecto perceptible sobre todo en el caso de los cuerpos especiales, la realidad es que muchos de los candidatos, singularmente los procedentes de familias acomodadas, han seguido un iter marcado por el fin de los estudios superiores y posterior concentración en preparar las pruebas del proceso selectivo, con lo que con carácter previo están enfrascados en escudriñar una realidad, con fuerte componente normativo, que reconocen sobre el papel. La situación no cambia una vez aprobado el proceso, pues el curso selectivo y las prácticas sirven más como instrumento de inducción y socialización que de formación práctica sobre el terreno social en que opera la Administración. La situación posterior, una vez adjudicado un puesto importante en el escalafón no mejora el asunto, puesto que suele primar el trabajo de oficina y relacional en el ámbito intra-administrativo, con lo que se habrá consolidado el déficit de realidad o desplazamiento de metas al que se refería Merton. El sistema selecciona altos funcionarios con sólida formación teórica, es verdad, pero al precio de otorgarles una pobre relación con la realidad social que, en muchos casos, no les abandonará en su carrera. Este aspecto, como se verá a continuación, ha estado muy presente en la reforma francesa, que cuestiona radicalmente el proceso selectivo y de carrera de sus élites administrativas, mientras que en España suelen contar con un respaldo complaciente por parte de los cuerpos (Ruano et al., 2014; Crespo, 2021).

\section{LA EXPERIENCIA FRANCESA: LA REFORMA DE LA LEY DE MAYO DE 2019 O LA SUPRESIÓN DE LA ENA}

\section{a) Contexto y diagnóstico}

Aunque en su discurso de investidura el presidente Macron ${ }^{7}$ no había hecho mención directa al abordaje de una reforma administrativa, lo cierto es que sí había mostrado su predisposición a afrontar de manera genérica aquellos grandes problemas que estaban generando parálisis y desconfianza en el país, y uno de esos factores, quizás el más importante, era la cuestión de las élites político-administrativas. La crítica a las élites, simbólicamente identificadas con su principal institución de reclutamiento y selección (la ENA), había destacado desde hacía años un alejamiento del ciudadano, una mayor predisposición a lo normativo y, a la postre, una mala inteligencia de los asuntos públicos. Lo que además se había puesto en evidencia en la gravedad de episodios recientes tales como la crisis de los chalecos amarillos, o la gestión de la pandemia del COVID-19. Para conocer la ambición reformadora del Presidente Macron sobre la alta función pública es útil acudir a sus intervenciones en las convenciones gerenciales del Estado, especialmente a la última, celebrada el 8 de abril de 2021; ello es así, tanto por el foro específico en que se hacen las declaraciones, como por la extensión y profundidad con que el presidente desgrana su pensamiento sobre el particular ${ }^{8}$.

En la última convención citada, se plantean algunas de las líneas maestras de la reforma, partiendo de reconocer que a pesar de que la Administración había estado a la altura durante el confinamiento provocado por la pandemia, sin embargo, las críticas seguían aflorando, puesto que las nuevas tecnologías usadas, habían facilitado el escrutinio inmediato y la comparación de la gestión con otros países.

En primer lugar, se reconoce que en la Administración francesa no se afrontan debidamente los grandes desafíos generales: globalización y mundialización de las políticas, desindustrialización, cambios territoriales, mayores desigualdades en razón de las mutaciones de un capitalismo abierto, el cambio climático y sus consecuencias, la aceleración de las crisis geopolíticas y sanitarias, etc.

En segundo lugar, se considera necesario adaptarse a uno de los factores que acelera los cambios, y que se cifra en las tecnologías disruptivas combinadas con la gestión de datos («Le Numérique»). Este factor modifica la manera de relacionarse con la Administración, los usos y procedimientos, pero también permite una mirada crítica con carácter inmediato y transparencia. Los actores públicos deben comprender que la

7 Puede consultarse en: https://www.elysee.fr/front/pdf/elysee-module-725-fr.pdf (fecha de acceso: 30 de septiembre de 2021).

8 Puede consultarse la intervención de 8 de abril de 2021 en: https://www.elysee.fr/front/pdf/elysee-module-17482-fr.pdf (fecha de acceso: 30 de septiembre de 2021). 
acción pública está sometida a una aceleración, transparencia y comparación permanentes, y que si bien ello puede generar una sensación de vértigo y malestar, también puede propiciar una respuesta adecuada que genere confianza y legitimidad.

Y en tercer lugar, y quizás por los cambios y desafíos citados, se constata que los ciudadanos desconfían de la acción pública y de sus agentes. Pero sea la desconfianza por la razón que sea, la situación pone en cuestión el consentimiento y la legitimidad de los actores públicos, la acción pública y, en definitiva, del sistema político. Lo que transformaría una crisis de adaptación al entorno en una crisis democrática. El presidente Macron, aunque reconoce este peligro, sin embargo expresa su confianza de que la crisis de la acción pública quizás sea una crisis de eficacia y de lejanía de la Administración respecto de los ciudadanos que una crisis de la democracia en sentido estricto, lo que invita a actuar. Ante ello, el presidente asume que el Estado francés, que fue quien creó la nación francesa, tiene una responsabilidad histórica y ejemplarizante, y debe intervenir en lo que se considera un cambio estructural, buscando que la Administración francesa y sus élites administrativas acompañen a la ciudadanía y se parezcan más a ella.

\section{b) La situación de la ENA}

El proyecto de creación del organismo que sustituirá a la ENA fue aprobado el 7 de junio de 2021, pero resulta interesante, antes de presentar las novedades del mismo, conocer por qué se creó ésta y cuál ha sido su evolución en los últimos años.

Puede ser útil, para entender su creación, conocer que a mediados de los años 30 del pasado siglo la alta función pública francesa planteaba un problema: estaba en manos de una casta de notables, su reclutamiento era antidemocrático y era contratada directamente por organismos de empleadores de ministerios o por concursos que eran concursos específicos para cada organismo. Muchos funcionarios no tenían el nivel apropiado ni reunían los requisitos básicos de de carácter técnico.

Se necesitaba una Administración reclutada con criterios estrictamente republicanos.

En 1945 se crea la ENA. Las primeras promociones que salen participan activamente en la reconstrucción tras la II Guerra Mundial. Al regreso de De Gaulle en 1958, la ENA se convertirá en un arma al servicio del poder, apoyando un gobierno fuerte, pero pronto aparecen las primeras críticas por parte de activistas de izquierdas, como Pierre Mendès, que acusa a la «gente de la ENA» como «odiosa de poder» y comienza a utilizarse el término «enarquía», un término peyorativo que hasta el diccionario termina incluyendo. Se critica ya entonces la falta de diversidad.

A mediados de los 80 , imbuidos quizás por el pensamiento neliberal imperante, ciertos alumnos comienzan a dudar si elegir trabajar para el ámbito público. Con el presidente Chirac se asentará la visión de la búsqueda de las puertas giratorias. La opinión pública asimila a los enarcas con ellas, carreras políticas fáciles y, a veces, con el tráfico de influencias. Una de las voces más mediáticas es la del sociólogo Pierre Bourdieu, crítico con lo que considera la alta y distante arrogancia de la burocracia.

Aunque hubo varios intentos previos de eliminar la famosa clasificación de salida (que permitía a los primeros de cada promoción elegir el alto cuerpo e Institución en que trabajar), en 2019, Macron, tras el fenómenos de los «chalecos amarillos» y la denuncia social del «sistema de élites» del país, asume que es necesario suprimir la ENA, llegando a subrayar que la clasificación de salida ha mantenido las mismas estructuras de siempre sin poder reformar nada. Con esta clasificación de salida (los detractores decían que se preparaba a los estudiantes para clasificarlos más que para formarlos ${ }^{9}$ ), se permitía a los quince alumnos más brillantes de una promoción elegir su plaza, ajenos muchas veces a la realidad y sintiéndose acomodados, y sin la obligación de asumir nuevos desafíos en su carrera. Otros autores relevantes han destacado que la ENA reproduce las élites, la burocracia y la centralización, así como su falta de adaptación a los desafíos del presente y del futuro, destacando entre ellos el ya citado Pierre Bourdieu ${ }^{10}$, junto a Jean-Pierre Chevénement ${ }^{11}$ y Michel Crozier ${ }^{12}$.

\footnotetext{
9 Por ejemplo, Olivier SABY, antiguo alumno, en su libro Promotion Ubu Roi, Flammarion Document, 2012.

10 Escribió junto a Jean-Claude Passeron Les Héritiers, les étudiants et la culture (Les éditions de minuit, 1964), destacando cómo los herederos de la cultura dominante son los que acceden a los centros formativos de élite en Francia.

11 Señaló, mediante el seudónimo Jacques Mandrin, la creación mediante la ENA de un sistema de mandarines burgueses en L'Enarchie (La table ronde, 1967).

12 Quien denuncia que las élites francesas son víctimas de un sistema educativo endogámico y conservador, lo que tiene como resultado una falta de adaptación a los desafíos presentes y futuros (véase, su libro La crisis de la Inteligencia. Ensayo sobre la incapacidad de las élites para reformarse, INAP, 1996).
} 
Sin embargo, el análisis impone un juicio más ponderado. La ENA, sin ser perfecta, ha cumplido en gran medida su misión: puso fin a la cooptación y al nepotismo de altos funcionarios y ha creado un elenco de egresados de altísimo nivel. Por otra parte, se ha luchado por la democratización ( $29 \%$ de los estudiantes becados), aunque algunos de estos estudiantes sostengan que el ascensor social está roto, con una fractura evidente entre los de la primera planta y la planta baja.

Eliminar a los enarcas parece una solución respaldada tanto en la derecha como en la izquierda; solo algunos enarcas sostienen que es un error suprimirla, sin explorar vías de reforma para corregir el elitismo y remarcando que hay que cambiar el debate, que lo que se necesita es reformar la democracia, que se necesita consultar más al francés, que todas las decisiones están centralizadas y eso hace que el ciudadano vea elitismo como lejanía del Estado en relación con su vida.

La ENA será reemplazada por un Instituto Nacional de Servicio Público (INSP) a partir del 1 de enero de 2022. El establecimiento estará ubicado en Estrasburgo y la idea es que pueda estar más abierto a la igualdad de oportunidades y su formación, más en conexión con la realidad social y el mundo académico.

\section{c) Las propuestas}

Para el presidente Macron, las respuestas al desafío de la reforma deben asentarse tanto en los principios republicanos tradicionales (interés general, lealtad, neutralidad), como en otros contemporáneos: proximidad, eficacia, simplicidad e innovación.

Cabe precisar, antes de especificar las propuestas, que la supresión de la ENA es uno de los cambios estrella de las mismas, pero que se inscribe dentro de un planteamiento más general y ambicioso, puesto que no solo afecta al sistema de reclutamiento y selección, sino también a la propia estructura de los colectivos que conforman la función pública (los cuerpos), y los itinerarios de carrera profesional. Como un objetivo principal de este estudio es reflexionar sobre la diversidad de la extracción de los altos funcionarios, nos centraremos especialmente en lo que se refiere al reclutamiento y selección, pero ello no obsta para que se haga un breve desarrollo de conjunto, basado en los tres pilares en que se quiere apoyar la reforma de la alta función pública: diversificar el reclutamiento, descompartimentar la estructura y dinamizar la carrera.

\section{Diversificar el reclutamiento y la selección}

No puede decirse que la élite administrativa francesa sea diversa. En efecto, el Rapport Thiriez (2020, pp. 10-11) explica pormenorizadamente como existe una sobrerrepresentación de las clases superiores en las grandes escuelas del servicio público, indicando que si bien un candidato procedente de una clase superior posee 1 posibilidad sobre 10 de acceder a la ENA, la situación empeora para los procedentes de "clases populares» (1 posibilidad sobre 43, para el concurso externo), situación que se agrava si se considera el diferente peso demográfico de cada colectivo. Por otra parte, también se produce un desequilibrio en la representación de género en favor del masculino, especialmente destacado en la Escuela Politécnica (solo $22 \%$ mujeres), y también notable en la ENA (32\% de candidatas aprobaron el acceso de media en el período 2010-2018). Esta tendencia se da también en los puestos de Director de Hospital y, sin embargo, solo en los concursos de Administradores Territoriales aprueban más mujeres que hombres (datos de 2017). Finalmente, otro problema es el casi monopolio parisino en la preparación de los concursos, sobre todo los que han pasado por Science Po Paris, que representan por ejemplo el $76 \%$ de los admitidos a concurso externo en la ENA, 2/3 de los admitidos a concurso en la Asamblea Nacional y Senado, o que 1/3 de los auditores de justicia han pasado por sus clases, etc. Además, el $40 \%$ de los admitidos a concurso han nacido en Paris, pero la «parisinización» se refuerza porque $2 / 3$ de los admitidos además han acabado un grado universitario en esta capital. Esto significa que hasta ahora ha sido muy conveniente estudiar en Paris para poder tener éxito en las pruebas de acceso, y que ello prefigura el perfil socioeconómico y territorial de los candidatos. Otro problema adicional, no exactamente de diversidad pero que incide en el reclutamiento y selección, es la disminución del atractivo para los jóvenes de la alta función pública, puesto que en los últimos años ha bajado el número de candidatos mientras que se incrementaba el número de plazas. 
Ante esta situación la reforma propone:

- Abrir el acceso a la alta función pública mediante contratos privados, que permita atraer a personal externo que puedan aportar recursos y experiencia a la función pública.

- La alta función pública debe parecerse a la sociedad, acabando con los determinismos y sesgos. Se crea el dispositivo Talentos para diversificar los perfiles de la función pública. Para ello, habrá 74 clases preparatorias de los concursos en todo el territorio de Francia ya en septiembre de 2021 (ver gráfico siguiente), con 1.700 plazas destinadas a estudiantes universitarios becados que han finalizado con grandes resultados una licenciatura (licence) o master. Con ello se pretende mejorar la diversidad de la alta función pública en términos territoriales y socioeconómicos.

Figura 1. Ubicación geográfica de LAS CLASES PRÉPAS tALENTS (2021-2022)
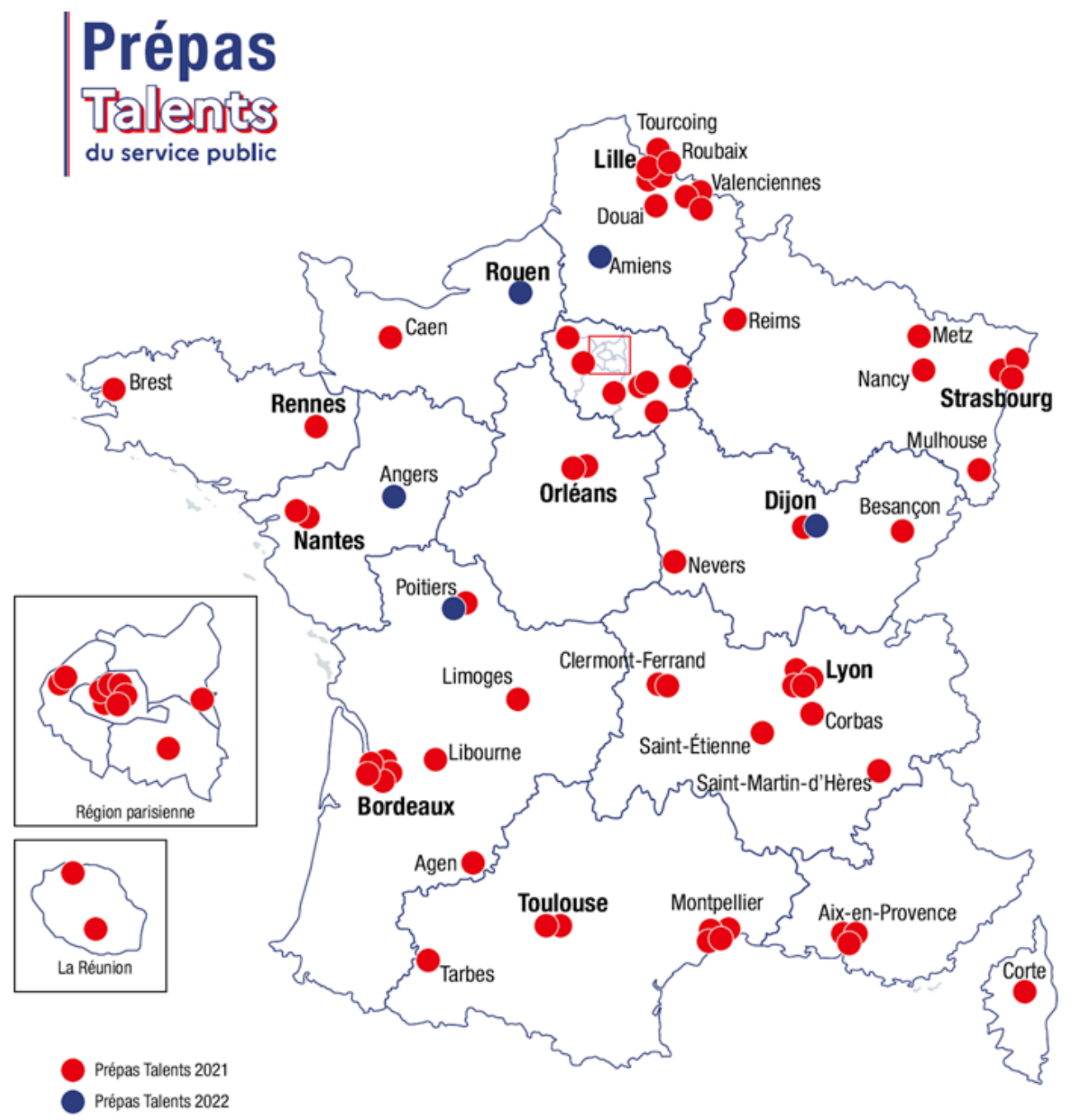

Fuente: https://www.transformation.gouv.fr/la-ministre/actualite/decouvrez-les-prepas-talents-pres-de-chez-vous (fecha de acceso: 1 de septiembre de 2021).

- Todos los altos funcionarios deberán trabajar inicialmente sobre el terreno, y en general, fuera de Paris. Ello afectará a su carrera.

- Supresión de la ENA, aunque a efectos de diversidad probablemente no era su existencia en sí la que generaba el sesgo, sino que el mismo procedía del sistema educativo y socioeconómico, junto con el diseño del proceso selectivo. Es decir, que en principio podrían haberse hecho cambios sin haber suprimido la ENA. Pero probablemente la talla cualitativa y cuantitativa de la reforma implica- 
ba un cambio tan profundo que conllevaba una refundación de la misma, que es lo que de alguna manera se hace con el Instituto Nacional de Servicio Público (INSP).

\section{La descompartimentación de la alta función pública}

Uno de los problemas de los modelos de carrera con una alta función pública cerrada en el acceso y en la salida, es la fuerte corporativización de las funciones administrativas y de los espacios organizativos, que con frecuencia lleva a una patrimonialización del interés público y a una resistencia al cambio. La reforma francesa tiene la ambición de modificar el status de los altos cuerpos, cuyos integrantes, se critica, están muy alejados de los ciudadanos, refugiados en sus oficinas parisinas, creando, aplicando, supervisando y sancionando el cumplimiento de normas sobre una ciudadanía que se siente abandonada por un colectivo sentido como ajeno y muchas veces hostil.

Las medidas para descompartimentarla son variadas. En primer lugar, el INSP formará a todos los aprobados de la alta función pública francesa en un tronco común, que perseguirá crear una cultura común y comprender los grandes problemas del momento. Dichos aprobados además formarán parte de un único cuerpo de salida, el de Administradores del Estado, y deberán pasar obligatoriamente un período trabajando sobre el terreno (se estima que de los 80 alumnos formados anualmente en la ENA, solo un $10 \%$ tomaba destino en la Administración desconcentrada, la más cercana a la realidad por proximidad y relación con el conjunto del territorio francés). La asignación de puestos funcionales vendrá después, pero ello implicará que previamente se «funcionalicen» los puestos, es decir, que se establezcan criterios para realizar catálogos funcionales, organizados en torno a factores comunes a los diversos puestos, de manera que se puedan crear carreras de manera lógica y en concordancia con las competencias que conlleva su desempeño. Por otra parte, la carrera y retribuciones del alto funcionario estará condicionada por las evaluaciones que reciba y su paso por diferentes puestos sobre el terreno. Además, se creará una Delegación Interministerial que acompañará al funcionario en el diseño de su carrera, junto a la creación de Viveros de Talento, mediante los cuáles se estimularán las promociones a medio y largo plazo. Por lo tanto, conceptos como proximidad, experiencia en el terreno, evaluación del desempeño y una nueva gestión del talento se configuran como factores clave de la reforma.

\section{Dinamizar las carreras de los altos funcionarios}

Uno de los problemas de los egresados de la ENA, sobre todo los que se ubicaban en los puestos de cabeza en la clasificación de cada año, es su asociación a una alta posición en la Administración, normalmente en los órganos centrales o en los cuerpos de élite de instituciones centrales, como el Tribunal de Cuentas. Lo que implicaba que personas jóvenes con carácter inmediato se veían insertos en una estructura en que los estímulos para progresar estaban limitados, además del sesgo que supone comenzar a adoptar decisiones respecto de la creación y aplicación de normativas sobre escenarios que rara vez se conocían en profundidad. Se interpreta que estas prácticas podrían afectar a la percepción por parte de la ciudadanía de la eficacia, ritmo y lejanía de la acción pública, y haber profundizado su desafección. Por eso, se toman las siguientes medidas:

- Salvo casos excepcionales, todos los altos funcionarios deberán trabajar períodos en la Administración desconcentrada, sobre el terreno.

- Se creará la Delegación Interministerial para los cuadros del Estado (Délégation interministérielle à l'encadrement supérieur de l'État).

- Posibilidades de salidas al sector privado y entradas desde el sector privado.

- Creación de Viveros de Talento.

- Establecimiento de un sistema de selección y promoción transparente.

- Apertura de un debate sobre la equidad interna y externa de los salarios.

- El INSP proporcionará formación a los altos funcionarios cuando desempeñen nuevas funciones o haya cambios significativos en las que desarrollan.

Finalmente, se ha incluido en la reforma, además de un elenco de estudios y consultan, un calendario preciso de su introducción, que supera el marco temporal que corresponde al presidente Macron, salvo reelección (2017-2022): 
DA. Nueva Época - N. ${ }^{8}$, diciembre 2021 - ISSN: 1989-8983 - DOI: https://doi.org/10.24965/da.i8.11022 - [Págs. 9-24]

¿Debe la alta función pública parecerse a la sociedad? Algunas enseñanzas de la reforma de la alta función pública en Francia

Jorge Crespo González

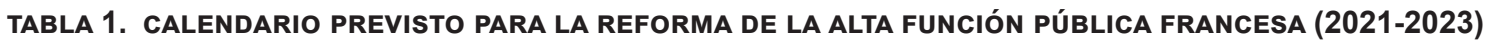

\begin{tabular}{|c|c|}
\hline FECHAS & MEDIDAS \\
\hline Desde 2021 & $\begin{array}{l}\text { - Reclutamiento (selección) mediante los concursos Talentos (Talents), para acceder al ISP y el } \\
\text { resto de escuelas del servicio público (INET, EHESP, ENAP, ENSP). } \\
\text { - Apertura de las clases preparatorias Talents. } \\
\text { - Creación del Ciclo de altos estudios del servicio público (CHESP) y admisión en su seno de los } \\
\text { cuadros superiores salidos de la alta función pública territorial, hospitalaria, de la magistratura y } \\
\text { del sector privado. } \\
\text { - Implantación de un tronco formativo común en las Escuelas de Servicio Público que forman a los } \\
\text { cuadros superiores de la función pública. } \\
\text { - Implantación de nuevos procesos de reclutamiento para algunos empleos creados por la reforma. } \\
\text { Establecimiento de una misión respecto de los Cuerpos Técnicos del Estado. }\end{array}$ \\
\hline En 2022 & $\begin{array}{l}\text { - Creación del Instituto nacional del Servicio Público (ISP). } \\
\text { - Creación de la Delegación interministerial para los cuadros superiores del Estado. } \\
\text { - } \text { Administradores Civiles. } \\
\text { - } \quad \text { Nuevo régimen común de indemnizaciones para los Administradores del Estado. } \\
\text { - } \quad \text { creación de estatutos de empleo, o con características propias, a favor de algunos de los empleos } \\
\text { - Implantación de evaluaciones realizadas por instancias colegiadas }\end{array}$ \\
\hline En 2023 & $\begin{array}{l}\text { - Final de reclutamientos (selecciones) en los demás cuerpos. } \\
\text { - } \quad \text { Primera promoción emanada del INSP }\end{array}$ \\
\hline
\end{tabular}

Fuente: https://www.transformation.gouv.fr/reforme-de-l-encadrement-superieur-de-l-etat/calendrier (traducción propia; fecha de acceso 30 de septiembre 2021).

\section{ENSEÑANZAS PARA EL CASO ESPAÑOL}

Los motivos que impulsan la reforma de la alta función pública francesa no son ajenos a nuestra alta función pública. En efecto, se comparte una buena parte del diagnóstico y del contexto que coadyuva a ese cambio necesario (mismos problemas globales, coincidencia básica de cultura administrativa, etc.), pero sin embargo hay aspectos que condicionan las respuestas dadas desde el Gobierno del Estado en cada uno de los países.

En primer lugar, la potencia de la institución de la «función pública» se ha desdibujado en nuestro país desde la aprobación del Estatuto Básico del Empleado Público, cambiando el ángulo de comprensión de la relación «funcionario respecto de la Administración», anteriormente basada en la función que debía garantizar (de ahí «funcionario»), y pasando ahora a ser un mero empleado público (que desempeña un empleo cuyo empleador es la Administración). Sobre la importancia institucional de la función pública ya nos había alertado Prats (1995) tempranamente y nos lo ha vuelto a recordar recientemente Jiménez Asensio $(2020)^{13}$.

En segundo lugar, y por las especificidades propias de nuestra articulación territorial, dicha institución (la de la «función pública») se ha cuarteado y dividido con las funciones públicas de las CC.AA, lo que hace que la alta función pública netamente del Estado sea la que se encuentra al servicio de la AGE y de los altos órganos constitucionales. Por otra parte, desde hace lustros se viene pidiendo una reforma de la alta función pública, especialmente para reconocer y regular adecuadamente la figura del Directivo Público Profesional, pero si bien se han permitido los debates y se han estimulado algunos estudios, finalmente la pobreza de las materializaciones prácticas es apabullante. Ese espacio de interpenetración entre política y alta administración que comparten en España el colectivo de los políticos y el de los integrantes de algunos cuerpos de élite se caracteriza por dos males endémicos: o la politización de la cúspide de la Administración, ya sea directamente por políticos con poca experiencia de gestión; o la corporativización a favor de unos pocos cuerpos de los puestos más destacados por su naturaleza directiva. Es decir, una alta función pública, con algunos matices, cerrada en la entrada y en la salida, politizada y corporativizada. Esto no puede dejar de

13 https://rafaeljimenezasensio.com/2020/02/22/la-institucion-de-la-alta-funcion-publica-como-politica-de-estado-reforma-enfrancia-algunas-lecciones/ (fecha de último acceso: 30 de septiembre de 2021). 
generar confusión entre las lógicas políticas y las directivas y de gestión, siendo los procesos de selección poco transparentes, desestimulando el talento y alentando el clientelismo político, lo que favorece las decisiones subóptimas, dada la primacía de su componente cortoplacista.

Un ejemplo reciente podría ilustrarse a través de los meritorios esfuerzos de prospectiva que el Gobierno del Estado ha impulsado a través de los documentos denominados España $2050^{14}$ y el Plan de Recuperación, Transformación y Resilencia ${ }^{15}$. En ellos, aunque se declara perseguir una mejora de la Administración y función pública, realmente se impone un interés por mejorar la eficacia y eficiencia del gasto o su consideración en tanto instrumento necesario para introducir los programas de inversión financiados total o parcialmente con fondos europeos; incluso se pretende señalar los beneficios de la potenciación de la digitalización de la Administración de cara al ciudadano, pero sin embargo se sigue sin otorgar a la alta función pública el rol estratégico e institucional que realmente tiene, por lo que algunos de los «olvidos» que se incluyen en dichos planes (como, por ejemplo, la introducción de una verdadera función pública directiva profesional) sean especialmente lacerantes.

En este marco, cabe retener varias enseñanzas del caso francés:

1. La función pública, y por supuesto la alta función pública, es una institución básica del Estado, y ello conlleva que una reforma ambiciosa de la misma deba asumirse como política de Estado.

2. Debe ser asumida e impulsada por las altas autoridades políticas del Estado: El Primer Ministro, el Gobierno y el Presidente o Jefe del Estado.

3. Debe incorporar un marco jurídico suficiente y eficaz, incardinado en un proyecto estratégico de cambio que considere los objetivos, pero también las condiciones de partida y los requerimientos del proceso de cambio.

4. Debe incorporar una infraestructura organizativa de apoyo, tanto para gestionar la nueva realidad, como para gestionar el cambio. En el caso de la reforma francesa, bajo el liderazgo del Presidente y del Primer Ministro, se ha trasladado la concreción de la reforma a la Ministra de Transformación y Función Pública (Ministre de Transformation et Fonction Publique). Además, la propia reforma incluye instrumentos de acompañamiento al nuevo sistema: la Delegación Interministerial, el Dispositivo Talents, etc.

5. Deben incorporarse mecanismos de difusión y socialización del cambio. Si se observa el caso francés, destaca la decidida voluntad del Presidente, implicado directamente en su implantación, tal como demuestra su participación en las convenciones gerenciales del Estado (conventions managériales de l'Etat) y su iniciativa de remitir una carta a los cuadros superiores del Estado en que, mediante 6 páginas, les traslada las causas y el objeto de la reforma, cómo les va a afectar y qué se espera de ellos. Y también el rol de la ministra de Transformación y Función Pública, quien realiza múltiples acciones de pedagogía del cambio, ya sea participando en foros políticos y profesionales, respondiendo a entrevistas de la prensa, e incluso preocupándose expresamente de la situación de los actuales alumnos de la ENA y de los candidatos que se encuentran en escuelas preparatorias, a los que ha dedicado vídeos explicativos específicos. Además, la web del ministerio ${ }^{16}$ ha desplegado un elenco de documentos que transmiten con claridad qué se pretende hacer, por qué, cómo afecta a los diferentes actores implicados, junto con un apartado completo de FAQS ${ }^{17}$.

6. Debe asociarse a los ciudadanos en las decisiones más importantes. El Presidente Macron reconoce que en su decisión han pesado los resultados del Grand Débat National y de la Convention Citoyenne. Uno de los principales objetivos de la reforma es precisamente acercar la ciudadanía al espacio de la alta función pública y viceversa.

7. Lo anterior sirve como sistema de orientación, difusión y control de la reforma.

8. Respecto de los aspectos técnicos que interesan a este trabajo, cabe señalar que se afronta valientemente la necesidad de modificar el sesgo socioeconómico y territorial que afecta a los altos funcionarios, mediante el dispositivo Talents y la reserva de cupos o concursos específicos para los candidatos procedentes de esta vía. Los ensayos previos realizados en Science Po París desde

14 Accesible en: https://www.lamoncloa.gob.es/presidente/actividades/Documents/2021/200521-Estrategia_Espana_2050.pdf (fecha de acceso: 30 de septiembre de 2021).

15 Accesible en: https://www.lamoncloa.gob.es/temas/fondos-recuperacion/Documents/30042021-Plan_Recuperacion_\%20 Transformacion_\%20Resiliencia.pdf (fecha de acceso: 30 de septiembre de 2021).

16 Ver: https://www.transformation.gouv.fr/reforme-de-l-encadrement-superieur-de-l-etat (fecha de acceso: 30 de septiembre de 2021)

17 Pueden consultarse en: https://www.transformation.gouv.fr/files/actualites/FAQ_reforme-haute-fonction-publique.pdf (fecha de acceso: 30 de septiembre de 2021). 
hace unos años, sin ser revolucionarios, han mostrado que se puede enriquecer los perfiles de los admitidos. Se han tomado decisiones para que la Administración, a través de su alta función pública, se parezca más a la sociedad francesa en el apartado subjetivo; pero también se ha pretendido que lo que se hace desde la Administración sea más parecido a lo que demanda la sociedad francesa, a través de las medidas de fortalecimiento de la Administración desconcentrada y la obligatoriedad para la alta función pública de trabajar sobre el terreno durante largos períodos de tiempo.

\section{CONCLUSIONES}

En este estudio hemos analizado la alta función pública, su noción e importancia desde el punto de vista político y administrativo, y la necesidad de incorporar diversidad a la misma para que los ciudadanos, con independencia de sus recursos, puedan tener un acceso equitativo no solo a los resultados de las acciones públicas, sino también al sistema político-administrativo del Estado.

Hemos definido la alta función pública en nuestro entorno como aquellos cuerpos integrados por funcionarios con alta cualificación y profesionalidad que desempeñan los puestos de mayor poder administrativo. Se ha constatado que tanto por sus funciones, por su proximidad al proceso decisorio y por considerarse un buen trampolín para acceder a la política electiva, es crítico el examen de las condiciones de reclutamiento y selección de este colectivo, de manera que los sesgos que incorporan (entre ellos la procedencia territorial o de carácter socio-económico) ubicarían la discusión no solo en un escenario técnico (¿el sistema selecciona realmente a los mejores?), sino de democracia (¿el sistema selectivo genera per se, o agrava, la situación de algunos estratos sociales en su relación con la política?).

En España hemos visto como la alta función pública del Estado, ilustrada fundamental en este estudio por el Cuerpo de Administradores Civiles del Estado, tiene varios sesgos. En lo que se refiere al territorial (procedencia de los integrantes de ese cuerpo) se constatan desequilibrios de infrarrepresentación de algunas regiones (Cataluña, Extremadura y Canarias) respecto de otras, siendo palmaria, a efectos de sobrerrepresentación la región de Madrid. Y sorprende que esta situación prácticamente no haya sufrido cambios en los últimos 50 años, creación del Estado Autonómico incluida. En lo relativo al sesgo socioeconómico, los estudios muestran cómo la alta función pública está conformada muy mayoritariamente por personas procedentes de entornos acomodados, lo que se ve facilitado además por el propio sistema selectivo que impone costes importantes a los candidatos, en términos formativos y de preparadores, además de la espera de varios años hasta el posible aprobado. También, aunque con matices, se encuentran sesgos en materia de género y discapacidad. A pesar de ello, en España poco se ha hecho por disminuir estos sesgos territoriales y socioeconómicos, salvo la toma de consciencia y alguna iniciativa eventual como la del Ministerio de Justicia, que ha ofrecido becas a opositores a Jueces, Fiscales y Secretarios Judiciales, a través del Centro de Estudios Jurídicos ${ }^{18}$, y la descentralización de algunas pruebas de acceso.

Sin embargo, en Francia en los últimos años se está emprendiendo una reforma muy ambiciosa de la alta función pública, en que se busca, entre otros objetivos, conseguir que la Administración se parezca a la sociedad francesa. En esa línea se han tomado decisiones de gran calado simbólico, como la supresión de la elitista ENA, y la aparición del ISP. Se trata de una refundación más que de una reforma, que pretende crear una alta función pública menos compartimentada (con una fuerte cultura de servicio público común), con carreras más atractivas y más diversa. Sobre este último aspecto, que es el que más interesa a este trabajo, cabe decir que la creación del Dispositivo Talents, y la dispersión de las clases de preparación del acceso a la alta función pública por toda la geografía francesa, parecen un instrumento poderoso para intentar disminuir el déficit existente de candidatos procedentes del medio rural y de clases modestas, pero todavía es pronto para conocer los resultados. En lo que concierne a la identificación de lo que hacen los altos funcionarios con las necesidades de la sociedad, el diseño de una carrera profesional que obliga a aterrizar sobre el terreno, saliendo forzosamente de Paris, junto a la creación de carreras externas al sector público (tanto de entrada, como de salida), podría tener efectos benéficos para la adaptación de las acciones públicas a los contextos reales, pero de nuevo es pronto para evaluar los resultados. Finalmente, Esta situación de enérgico cambio

18 Curiosamente las ayudas a posibles candidatos u opositores han desaparecido en los últimos años, tal como se muestra en la última memoria de la institución, del año 2020, aunque aparecen algunos convenios de colaboración con CC.AA para preparar cursos selectivos y actividades formativas de su interés. Dicha memoria puede consultarse en: https://www.cej-mjusticia.es/sites/default/files/ adjuntos/2021-03/3.MEMORIA\%202020\%20CEJ.pdf (fecha de acceso: 30 de septiembre de 2021). 
DA. Nueva Época - N. ${ }^{\circ}$ 8, diciembre 2021 - ISSN: 1989-8983 - DOI: https://doi.org/10.24965/da.i8.11022 - [Págs. 9-24]

¿Debe la alta función pública parecerse a la sociedad? Algunas enseñanzas de la reforma de la alta función pública en Francia

Jorge Crespo González

contrasta con la situación de apatía, escepticismo e inercia que impera sobre la reforma de alta función pública española, de quien puede discutirse que represente a la sociedad en lo que es, y en lo que hace.

\section{REFERENCIAS BIBLIOGRÁFICAS}

Alvárez Alvárez, J. (1980). El origen geográfico de los funcionarios españoles. Instituto Nacional de Administración Pública - INAP.

Arenilla Sáez, M. y Delgado Ramos, D. (2019). Una nueva función pública que fortalezca la confianza en las instituciones públicas. Revista Vasca de Gestión de Personas y de Organizaciones Públicas, 16, 36-53. https:// apps.euskadi.eus/z16-a5app2/es/t59auUdaWar/R3/verArticulo? numejem=16\&tipo=R\&seccion=51\&correlativo=3\& contenido $=3 \&$ locale $=\mathrm{es}$

Arenilla Sáez, M. (2016). Naturaleza política y función social de la Administración Pública [Laudatio pronunciada en el acto de investidura de D. Mariano Baena del Alcázar como doctor honoris causa por la Universidad Rey Juan Carlos de Madrid, el día 9 de septiembre de 2016]. La Administración al día. Último acceso el 30 de junio de 2021 en: http://laadministracionaldia.inap.es/noticia.asp?id=1506602

Arenilla Sáez, M. (2014). Estudio introductorio. En J. M. Ruano de la Fuente (dir.), J. Crespo González y C. Polo Villar, Los funcionarios ante el espejo. Análisis del ciclo de la selección de personal en la Administración General del Estado. Instituto Nacional de Administración Pública - INAP.

Baena del Alcázar, M., Garrido, L., Pizarro, N. (1984). La élite española y la presencia en ellas de los burócratas. Documentación Administrativa, 200, 73-131. https://doi.org/10.24965/da.vi200.4761

Baena del Alcázar, M. (1988). Curso de la Ciencia de la Administración (Vol. I, 2. ${ }^{a}$ ed.) Tecnos.

Baena del Alcázar, M. (1999). Élites y conjuntos de poder en España (1939-1992): un estudio cuantitativo sobre Parlamento, Gobierno y gran empresa. Tecnos.

Beltrán, M. (1977). La élite burocrática española. Fundación Juan March - Ariel.

Carabaña, J. y Lamo de Espinosa, E. (2008). La élite burocrática y la movilidad social. En G. Meil y C. Torres (coords.). Sociología y realidad social: libro homenaje a Miguel Beltrán Villalva (pp. 391-424). Centro de Investigaciones Sociológicas.

Castillo Blanco, F., (2020). Un sistema de empleo distópico. ¿Seria necesario reformar el EBEP? Documentación Administrativa, 7, 8-32. https://doi.org/10.24965/da.i7.10889

Crespo González, J. (2021). Inclusión y origen geográfico la alta función pública del Estado español. Revista de Gestión Pública, 9(2), 197-230. https://doi.org/10.22370/rgp.2020.9.2.2923

Crespo González, J. (2020). Alta función pública y directivos públicos. En G. Pastor y B. Aldeguer (2020). Democracia, Gobierno y Administración Pública contemporánea (pp. 368-386). Tecnos.

Crespo González, J. (2018). Democracia y alta función pública en el marco intergubernamental del Estado español. Gestión y Análisis de Políticas Públicas, 19, 17-33. https://doi.org/10.24965/gapp.v0i19.10512

Crespo González, J. (2017). Coordinación intergubernamental en España vista por la alta función pública del Estado. Política y Sociedad, 54(2), 481-508. https://doi.org/10.5209/POSO.52199

Crespo González, J. (2015). Pluralismo geográfico del origen de los altos funcionarios de la Administración general del Estado. En E. González García, A. García Muñiz, J. García Sansano y L. Iglesias Villalobos (coords.), Mundos emergentes: cambios, conflictos y expectativas (pp. 768-782). Asociación Castellano-Manchega de Sociología. https://eprints.ucm.es/id/eprint/35666/

Del Pino, E. y Subirats, J. (eds.) (2021). Las administraciones ante los riesgos sociales y globales. Instituto Nacional de Admnistración Pública - INAP.

Del Pino, E. y Colino, C. (2021). ¿Qué sabemos sobre cómo reformar la Administración?: Contenidos, capacidad y trayectorias. En E. Del Pino y J. Subirats (eds.), Las administraciones ante los riesgos sociales y globales. Instituto Nacional de Admnistración Pública - INAP.

Genieys, W. (2004). Las élites españolas ante el cambio de régimen político. Lógica de estado y dinámicas centroperiferia en el siglo XX [monografía, 209] (D. Beltrán, trad.). Centro de Investigaciones Sociológicas - CIS.

Lins de Lessa, F. (2011). Acceso igualitario a la función pública. Editorial Juruá.

Linz, J., De Miguel, A. (1968). La élite funcionarial española ante la reforma administrativa. En AA.VV, Sociología de la administración pública española. Centro de Estudios Sociales de la Santa Cruz del Valle de los Caídos.

Luxan Meléndez, J. M. (2019). La ruptura del contrato de lealtad. Evolución del empleo científico-técnico en el sector público español. Cuadernos de Gobierno y Administración Pública, 6(2), 71-91. https://doi.org/10.5209/cgap.65911

Lynn, N. B. y Wildavsky, A. (comps.) (1999). Administración Pública. El estado actual de la disciplina. Fondo de Cultura Económica.

Areses Vidal, X., Blanco Gaztañaga, C., Colmenares Soto, P., Fernández Palomares, E., Gallego Torres, A., Gómez de Villalobos, G., İñiguez Hernández, S., Labrada Tellado, M. ${ }^{a}$ L., Mapelli Marchena, C., Pastor Sainz-Pardo. I., Souto Alonso, J. (2017). Nuevos tiempos para la función pública. Propuestas para atraer y desarrollar el talento en la Administración General del Estado. Instituto Nacional de Administración Pública - INAP. 
DA. Nueva Época - N. ${ }^{8}$, diciembre 2021 - ISSN: 1989-8983 - DOI: https://doi.org/10.24965/da.i8.11022 - [Págs. 9-24]

¿Debe la alta función pública parecerse a la sociedad? Algunas enseñanzas de la reforma de la alta función pública en Francia Jorge Crespo González

Merton, R. K. (1964). La Estructura Burocrática y la Personalidad. En R. K. Merton, Teoría y Estructuras Sociales (pp. 275-286). Fondo de cultura económica.

OCDE (2015). Panorama de las administraciones públicas 2015 [Goverment at Glance 2015]. OECD - INAP. https:// doi.org/10.1787/11d6485e-es

OCDE (2014). España: de la reforma de la Administración a la mejora continua [Informe de la OCDE sobre gobernanza pública en España]. OECD - INAP. https://doi.org/10.1787/9789264234192-es

OCDE (2009). Panorama de las administraciones públicas 2009 [Goverment at Glance 2009]. OECD - INAP. https:// doi.org/10.1787/9789264091412-es

Olías de Lima, B. (coord.) (2001). La nueva gestión pública. Prentice-Hall.

Olías de Lima, B. (coord.) (1995). La gestión de recursos humanos en las Administraciones Públicas. UCM - Editorial Complutense.

Panebianco, A. (1995). Las Burocracias Públicas. En G. Pasquino (comp.), Manual de Ciencia Política (pp. 365-410). Alianza Editorial.

Prats i Català, J. (1995). Los fundamentos institucionales del sistema de mérito: la obligada distinción entre función pública y empleo público. Documentación Administrativa, 241-242, 11-59. https://doi.org/10.24965/ da.v0i241-242.5394

Rodríguez Teruel, J., Jerez Mir, M. y Real-Dato, J. (2019). Las élites políticas en España: quiénes son, cómo son, qué hacen. En J. Montabes y A. Martínez (eds.), Gobierno y política en España (pp. 585-614) [colección Ciencia Política, 78]. Tirant lo Blanch.

Rodríguez Teruel, J. (2011). Los ministros de la España democrática: reclutamiento político y carrera ministerial de Suárez a Zapatero, 1976-2011. Centro de Estudios Políticos y Constitucionales.

Román Masedo, L. (1997). Funcionarios y función pública en la transición española [Cuadernos y debates, 69]. Centro de Estudios Políticos y Constitucionales.

Ruano de la Fuente, J. M. (dir.), Crespo González, J. y Polo Villar, C. (2014). Los funcionarios ante el espejo. Análisis del ciclo de selección de personal del Estado. Instituto Nacional de Administración Pública - INAP.

Sánchez Morón, M. (2011). El empleo público en España: problemas actuales y retos futuros. En R. Jiménez Asensio (ed.), El empleo público en Aragón y tendencias de futuro (pp. 19-28). Gobierno de Aragón.

Thiriez, F. (30 de enero de 2020). Mission Haute Fonction Publique. Propositions [Rapport Thiriez]. Último acceso el 30 de septiembre de 2011 en https://www.gouvernement.fr/sites/default/files/document/document/2020/02/rapport de_m._frederic_thiriez_-_mission_haute_fonction_publique_-_18.02.2020.pdf 